\title{
CHAMP climate data based on the inversion of monthly average bending angles
}

\author{
J. Danzer ${ }^{1}$, H. Gleisner ${ }^{2}$, and S. B. Healy ${ }^{3}$ \\ ${ }^{1}$ Wegener Center for Climate and Global Change (WEGC), University of Graz, Graz, Austria \\ ${ }^{2}$ Danish Meteorological Institute (DMI), Copenhagen, Denmark \\ ${ }^{3}$ European Centre for Medium-range Weather Forecasts (ECMWF), Reading, UK \\ Correspondence to: J. Danzer (julia.danzer@uni-graz.at)
}

Received: 10 July 2014 - Published in Atmos. Meas. Tech. Discuss.: 29 July 2014

Revised: 31 October 2014 - Accepted: 5 November 2014 - Published: 2 December 2014

\begin{abstract}
Global Navigation Satellite System Radio Occultation (GNSS-RO) refractivity climatologies for the stratosphere can be obtained from the Abel inversion of monthly average bending-angle profiles. The averaging of large numbers of profiles suppresses random noise and this, in combination with simple exponential extrapolation above an altitude of $80 \mathrm{~km}$, circumvents the need for a "statistical optimization" step in the processing. Using data from the USTaiwanese COSMIC mission, which provides $\sim 1500-2000$ occultations per day, it has been shown that this averageprofile inversion (API) technique provides a robust method for generating stratospheric refractivity climatologies.

Prior to the launch of COSMIC in mid-2006, the data records rely on data from the CHAMP (CHAllenging Minisatellite Payload) mission. In order to exploit the full range of available RO data, the usage of CHAMP data is also required. CHAMP only provided $\sim 200$ profiles per day, and the measurements were noisier than COSMIC. As a consequence, the main research question in this study was to see if the average bending-angle approach is also applicable to CHAMP data.

Different methods for the suppression of random noise - statistical and through data quality prescreening - were tested. The API retrievals were compared with the more conventional approach of averaging individual refractivity profiles, produced with the implementation of statistical optimization used in the EUMETSAT (European Organisation for the Exploitation of Meteorological Satellites) Radio Occultation Meteorology Satellite Application Facility (ROM $\mathrm{SAF}$ ) operational processing.
\end{abstract}

In this study it is demonstrated that the API retrieval technique works well for CHAMP data, enabling the generation of long-term stratospheric $\mathrm{RO}$ climate data records from $\mathrm{Au}-$ gust 2001 and onward. The resulting CHAMP refractivity climatologies are found to be practically identical to the standard retrieval at the DMI (Danish Meteorological Institute) below altitudes of $35 \mathrm{~km}$. Between 35 and $50 \mathrm{~km}$, the differences between the two retrieval methods started to increase, showing largest differences at high latitudes and high altitudes. Furthermore, in the winter hemisphere high-latitude region, the biases relative to ECMWF (European Centre for Medium-range Weather Forecasts) were generally smaller for the new approach than for the standard retrieval.

\section{Introduction}

Global Navigation Satellite System Radio Occultation (GNSS-RO) receivers onboard low-Earth-orbit (LEO) satellites have provided a nearly continuous global data record on the state of the atmosphere since the launch of the German CHAMP (CHAllenging Mini-satellite Payload) satellite in 2001. During its 7-year lifetime CHAMP provided, on average, around 200 occultations per day. With the launch of the six-satellite constellation FORMOSAT-3/COSMIC (henceforth referred to as COSMIC) (Constellation Observing System for Meteorology, Ionosphere, and Climate) in mid-2006, the number of occultations per day increased by an order of magnitude to more than 2000. A number of research satellites further add to the RO data records, and in late 2006 the 
first in a planned series of European polar-orbiting Metop satellites carrying RO instruments became operational.

GNSS-RO is likely to play an increasingly important role in climate research and climate monitoring, particularly for the upper troposphere and stratosphere. The GNSS-RO data provide accurate geophysical information with a high vertical resolution at altitudes from around 5 to $40 \mathrm{~km}$. A decisive advantage compared to other measurement techniques is that RO data from different instruments and missions can be combined without intercalibration of the data records. This is a consequence of the fact that the primary observables are phase shifts, or time differences, rather than radiances.

The observed phase shifts of the GNSS carrier wave during an occultation are processed to ray bending angles without the explicit use of any a priori information. However, the retrieval of more traditional atmospheric variables from the measurements requires the extrapolation of the bendingangle profile to infinity using an a priori model of bending angles. The first step in that retrieval is the computation of a refractivity profile from the bending-angle profile using an Abel transformation, where extrapolation to infinity is required due to the upper limit in the Abel integral (Eq. 1). The observed bending-angle profile is limited in altitude by a decreasing signal-to-noise ratio - the observed bendingangle values fall off exponentially, while the dominating measurement errors are relatively constant with height. For the current generation of $\mathrm{RO}$ instruments, and the processing schemes used operationally, the observed bending-angle values and the errors are of roughly the same magnitude near $\sim 60 \mathrm{~km}$. Above that, the errors are larger than the observed bending angles.

To handle the exponentially decreasing signal-to-noise ratios, the extrapolation step and the merging with the a priori bending-angle information are often combined with a smoothing of the retrieved bending angles over an extended vertical interval, usually above $\sim 40 \mathrm{~km}$. This processing step is referred to as "statistical optimization" (SO). The "optimized" bending-angle profiles are then used to retrieve refractivity profiles, and, from these, profiles of temperature, geopotential height and pressure are derived. Different approaches to the statistical optimization have been described in some detail by, e.g., Gorbunov (2002); Lohmann (2005); and Gobiet and Kirchengast (2004). Specific details of implementations at various GNSS-RO processing centers have been described by Ho et al. $(2009,2012)$, while the SO scheme used at the ROM SAF (Radio Occultation Meteorology Satellite Application Facility) is outlined in Lauritsen et al. (2011). The errors introduced by the statistical optimization are difficult to characterize and quantify. Furthermore, recent studies within the ROtrends Working Group have shown that the upper-level bending-angle initialization may be a source of structural uncertainties for the climate data products (Ho et al., 2012; Steiner et al., 2013). The different methods and background information used in the SO of processing centers leads to differences in the climate data products, mainly above $25 \mathrm{~km}$.

Climatologies of the geophysical parameters, e.g., zonally gridded monthly means, are commonly derived by binning and averaging the individually retrieved, statistically optimized profiles. However, recent studies have used an alternative processing approach, which circumvents the statistical optimization step and has a clear - but weak - dependency on the assumed a priori. Ao et al. (2012) and Gleisner and Healy (2013) independently described the use of averaged bending angles instead of individual profiles. In this averageprofile inversion (API) technique, the required suppression of random noise is obtained through averaging a large number of profiles rather than smoothing and merging with a background. The feasibility of this approach was demonstrated with COSMIC data, which provides $1500-2000$ profiles per day. Furthermore, preliminary investigations also showed that the method is relatively robust when face with a large reduction of data numbers - a random removal of $85 \%$ of the data gave very small differences in the mean refractivities below $40 \mathrm{~km}$ (Gleisner and Healy, 2013). However, it remained unclear whether the API approach could be applied to the period from 2001 to 2006 when only CHAMP was available. CHAMP only provided $\sim 200$ profiles per day, and they were considerably noisier than the COSMIC and Metop data available from the latter half of 2006 and onward. Any method used to generate RO climate data records must be able to handle this situation. Therefore, the present study investigates the applicability of the API method during the CHAMP era. The suppression of noise by averaging and the role of quality control procedures are also investigated.

Furthermore, it has been shown that the bending-angle initialization used until recently in the standard retrieval scheme at the DMI (Danish Meteorological Institute), the MSIS climatology (Mass Spectrometer and Incoherent Scatter Radar), may exhibit biases during winter conditions at high altitudes and high latitudes (Steiner et al., 2013). Hence, one of the research questions is whether the initialization can be improved for climate data using the new average bending-angle approach. Currently, at the level of single refractivity profiles, the DMI is addressing this existing limitation by algorithm improvements and by using the so-called BAROCLIM spectral model (Bending Angle Radio Occultation Climatology) as a priori information (Foelsche and ScherllinPirscher, 2012; Scherllin-Pirscher, 2013; Scherllin-Pirscher et al., 2014) for the SO. In this study we concentrate especially on the high-latitude and high-altitude region, comparing the new averaging approach to the standard approach.

The data used in this study are described in Sect. 2.1. Section 2.2 describes the processing of the data to refractivity. An analysis of averaged bending angles is given in Sect. 3, while the main results of API-processed bending angles are presented in Sect. 4. The discussion and conclusions are given in Sect. 5. 


\section{Data sets and method}

\subsection{Data sets}

The study focuses on occultation events observed by the CHAMP single-satellite mission from September 2001 until September 2008. At the UCAR/CDAAC database (University Corporation for Atmospheric Research/COSMIC Data Analysis and Archive Center), excess phase profiles and precise orbit information were retrieved and further processed into bending-angle and refractivity profiles at the ROM SAF, which is an RO processing center under EUMETSAT (European Organisation for the Exploitation of Meteorological Satellites) and hosted by the DMI; this was done using the ROPP (Radio Occultation Processing Package) software as the DMI retrieval package.

In this study, the bending-angle and refractivity profiles were analyzed on a monthly basis for $10^{\circ}$ zonal bins, leading to between about 3500 and 5500 profiles per month in the case of CHAMP data and about 50000 to 65000 profiles per month for COSMIC data, after several steps of quality screening (Gorbunov et al., 2006).

Furthermore, colocated ECMWF (European Centre for Medium-range Weather Forecasts) refractivity and bendingangle profiles from ECMWF analysis data were studied on $10^{\circ}$ latitudinal bins and used as a reference for the monthly mean profiles. The analysis data fields used were studied on a T42L91 resolution, since the T42 horizontal resolution matches the resolution of RO data $(\sim 300 \mathrm{~km})$. Until January 2006, they are given on 60 vertical levels (L60); after that the ECMWF switched to 91 vertical levels (L91). Furthermore, in December 2006, the ECMWF started to assimilate GPS RO data into the analysis fields.

\subsection{Method}

The production of geophysical parameters from GNSS RO measurements requires a retrieval chain described in detail by Kursinski et al. (1997). In the retrieval, an Abel transformation occurs, relating the refractive index $n$ to the bendingangle $\alpha$ through

$\ln n(x)=\frac{1}{\pi} \int_{x}^{\infty} \frac{\alpha(a)}{\sqrt{a^{2}-x^{2}}} \mathrm{~d} a$,

where $a$ is the impact parameter and $x=n r$, with $r$ being the radius of a point on the ray path. The integral over infinity poses a problem, since observational data has a limit in altitude to about $80 \mathrm{~km}$. Hence an extrapolation to infinity becomes necessary. Furthermore, the signal-to-noise ratio increases with decreasing altitude. One widely used approach is to replace or merge the noisy observed bending angles with bending angles from a climatological model, often in combination with more or less complex smoothing filters. This is referred to as SO, where a statistically optimized bending an- gle is obtained, i.e.,

$\alpha=\alpha_{\mathrm{b}}+\mathbf{K}\left(\alpha_{\mathrm{O}}-\alpha_{\mathrm{b}}\right)$,

yielding the optimized bending-angle $\alpha . \alpha_{\mathrm{O}}$ and $\alpha_{\mathrm{b}}$ are the observed and the background bending angle, respectively. $\mathbf{K}$ is the gain matrix, which can be written in terms of error covariance matrices for the observed and background bendingangle profiles. Using the statistically optimized bending angle for the Abel transformation, refractivity profiles can be calculated. From refractivity other geophysical variables, such as density, pressure and temperature, are retrieved (for a more elaborated description, see, e.g., Kursinski et al., 1997). When comparing the results of different processing centers, discrepancies in the atmospheric parameters arise mainly through the choice of the gain matrix and the background information (Ho et al., 2012; Steiner et al., 2013).

One of the most widely used sources of a priori background information has been the MSIS climatology. Until recently, this was also the standard at DMI. In this study it is used in an SO scheme based on optimal linear combination (OLC) devised by Gorbunov (2002). It has been pointed out by, e.g., Gobiet and Kirchengast (2004) and Foelsche et al. (2008) that the use of MSIS climatology as a priori may cause problems at high latitudes and high altitudes, particularly during winter. There may be no adequate profile in MSIS that fits the very cold conditions, and the best fitting profiles may simply be too warm.

In this study, zonal monthly mean bending-angle climatologies were first built and then forwarded through the Abel transformation in order to obtain zonal monthly refractivity climatologies (Ao et al., 2012; Gleisner and Healy, 2013). The averaged bending-angle CHAMP data were used up to an altitude of $80 \mathrm{~km}$. Above that an extrapolation of the averaged bending-angle profiles to infinity was still necessary, due to the upper boundary of the Abel integral (see Eq. (1)). An exponential extrapolation with a constant scale height of $7.5 \mathrm{~km}$ was used, as in Gleisner and Healy (2013). Furthermore, the sensitivity at the assumed scale height was tested, using scale heights of 6 and $9 \mathrm{~km}$. The impact on the refractivity below values of $40 \mathrm{~km}$ was found to be less than about $0.02 \%$.

\section{CHAMP bending-angle averages}

As a primary investigation, monthly average CHAMP bending angles within $10^{\circ}$ latitude bins were built analogously to the average COSMIC bending angles studied in Gleisner and Healy (2013). Figure 1 shows differences between the CHAMP average bending angles and ECMWF bending angles similarly constructed from colocated ECMWF profiles, using means and medians, respectively, and with/without the extra quality control described in Sect. 3.1. Below $50 \mathrm{~km}$, random errors resulting from ionospheric residuals, instrumental errors and neutral-atmosphere variability are sup- 

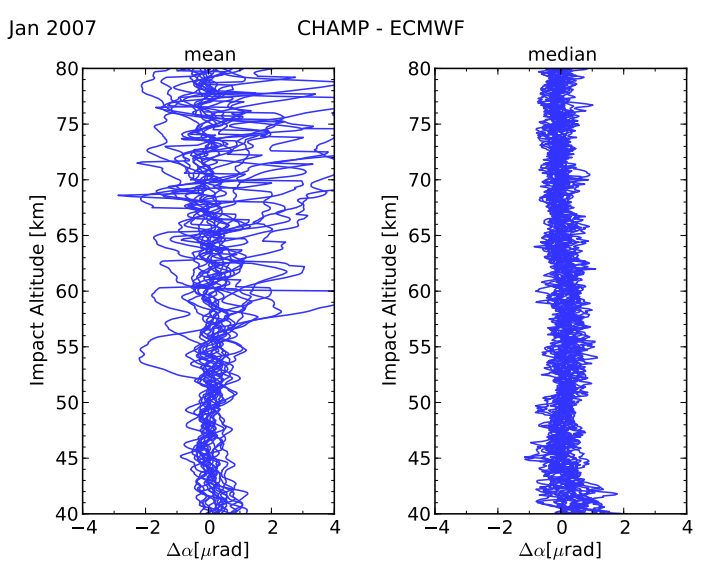

(a) Standard QC

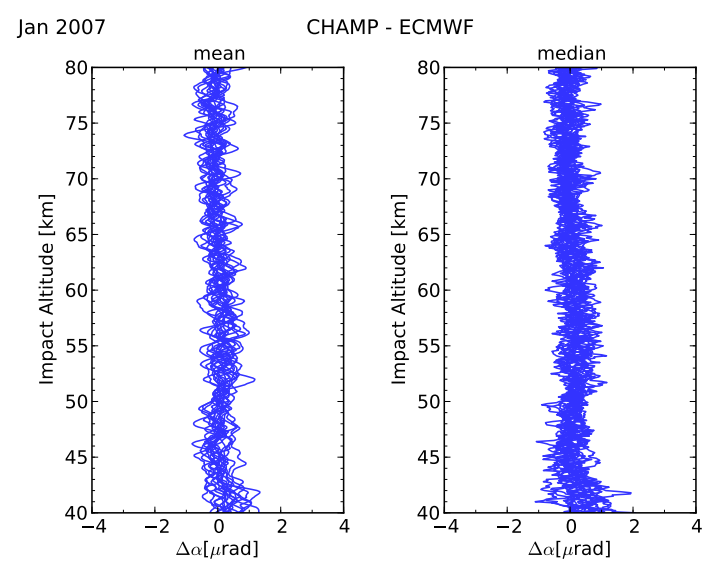

(b) New QC

Figure 1. Difference between observed bending-angle means and medians on the one hand and colocated ECMWF analysis data on the other, comparing the results with standard Qualitiy Control (QC) to the results where an additional bending-angle QC has been applied.

pressed due to averaging over many profiles within a bin, and the mean value is a smoother estimate compared to the median value. Above that, the mean bending-angle profiles show large-scale wiggles (Fig. 1a), leading to differences in the reference profile larger than $\pm 2 \mu \mathrm{rad}$. The median bending-angle profiles do not show such large-scale variations; however, they suffer from small-scale variations, which recommends the mean instead of the median bending angle as an average estimate below altitudes of about $50 \mathrm{~km}$.

\subsection{Bending-angle quality control}

In the processing from raw data to single bending-angle profiles, the DMI uses several screening steps in their quality control, in which data is partly or totally rejected (Gorbunov et al., 2006). However, regarding the increased noise in the usage of CHAMP satellite data compared to COSMIC satellite data, we decided to investigate more thoroughly the single bending-angle profiles contributing to the monthly mean zonal bending-angle estimates.

The left-hand side of Fig. 2 shows all single profiles contributing to the respective mean bending-angle profile for a zonal bin at a mean latitude of $5^{\circ}$. Obviously, single bendingangle profiles with values larger than $\pm 50 \mu \mathrm{rad}$ contribute to the estimate of the average bending-angle profile (blue line). Furthermore, the mean bending angle shows, dependent on altitude, a large standard deviation (green line). Performing the same analysis systematically on other zonal bins presents large bending-angle outliers contributing to the mean values of the single bins. Based on this result, we decided to perform an outlier rejection similar to the discussion in the ROM SAF report by Foelsche and Scherllin-Pirscher (2012). In an altitude range between 50 and $80 \mathrm{~km}$, they decided to reject all bending-angle profiles which are smaller than $-40 \mu \mathrm{rad}$ or larger than $40 \mu \mathrm{rad}$ from their BAROCLIM climatology.
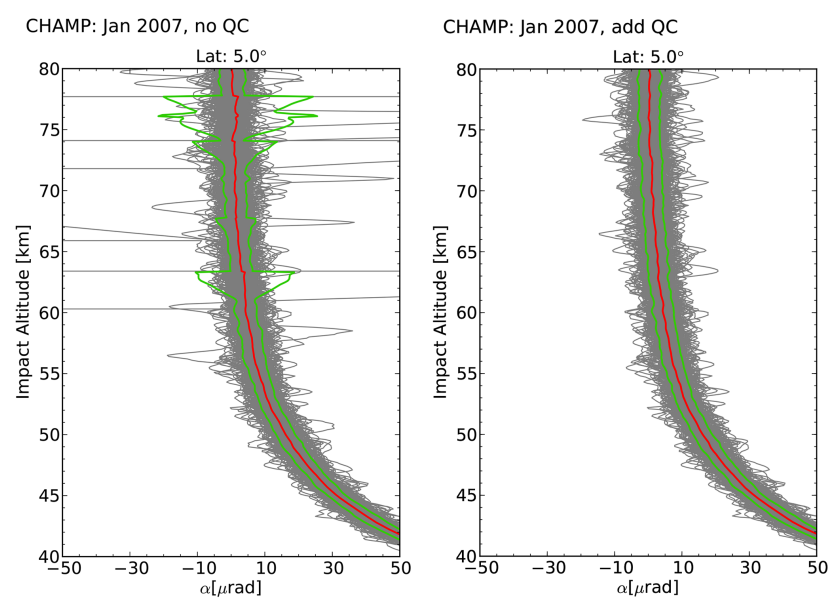

Figure 2. The left-hand plot shows all observational bending-angle profiles for the northern fundamental bin at mean latitude $5^{\circ}$, while the right-hand shows the same but outliers $\pm 30 \mu \mathrm{rad}$ have been rejected. The blue line represents the mean value of all profiles for each bin, the green line its standard deviation.

In this study we decided to reject an entire bending angle if single values of the profile were smaller or larger than $\pm 30 \mu \mathrm{rad}$ in the altitude region between 50 and $80 \mathrm{~km}$. To put this rejection criterion in context, the global, climatological average bending angle at $50 \mathrm{~km}$ is about $15 \mu \mathrm{rad}$. For the CHAMP satellite, we usually find between about 4000 up to 5000 profiles per month after applying the initial quality control used at the DMI. The additional bending-angle quality control (QC) reduces this number of profiles per month to a value of about 100 up to 400 profiles.

The right-hand side of Fig. 2 shows the result of the contributing single bending-angle profiles when an additional outlier rejection has been applied, studying the same mean 
latitude bin as before. Obviously profiles with large variations are eliminated, resulting in a smoother bending-angle estimate and a smaller standard variation. Figure $1 \mathrm{~b}$ shows the differences in the mean and median bending-angle estimates for January 2007 relative to the colocated ECMWF reference profiles for the case of an additionally applied QC. Clearly, rejecting the outliers removes large-scale wiggles in the mean bending-angle climatology, while the median bending angles are almost unaffected by the new approach.

Gleisner and Healy (2013) used a combination of mean and median values to estimate the average bending-angle values. Up to an altitude of $50 \mathrm{~km}$, they employed the mean bending angle; between the altitude range of 50 to $60 \mathrm{~km}$, they performed a simple linear combination between mean and median; and above $60 \mathrm{~km}$ only the median bending angle was used. In this follow-up study, we also used a linear combination of mean and median for averaged CHAMP bending angles. In an initial investigation, we studied the relative differences of mean-median combinations to the colocated ECMWF reference profiles, using a transition region between 50 to $60 \mathrm{~km}$ in a first attempt and comparing meanmedian combinations where additional quality control was applied to combinations without additional quality control. For the case without extra QC, some of the monthly meanmedian bending-angle estimates still exhibited, sometimes large, deviations from the smooth ECMWF reference profile, whereas averaged bending angles where an additional prior outlier rejection had been applied showed comparably small differences. Consequently, this further encouraged the idea of applying an additional QC to the single bending-angle profiles before calculating bending-angle means.

Furthermore, the sensitivity of employing different transition regions has been tested on the averaged bending-angle profiles. Transition regions between 45 and 55, 50 and 60, and 55 and $65 \mathrm{~km}$ with and without outlier rejection have been analyzed. Subsequently studying the Abel-processed averaged refractivity profiles, the results showed differences of less than $0.1 \%$ below altitudes of $45 \mathrm{~km}$ when comparing the transition regions. Hence, we decided to use a transition region between 50 and $60 \mathrm{~km}$ for mean-median bendingangle combinations, as used previously with the COSMIC data.

\section{CHAMP refractivity averages}

Zonal monthly mean refractivity profiles were computed for CHAMP data using the new average-profile inversion as well as the standard single-profile inversion.

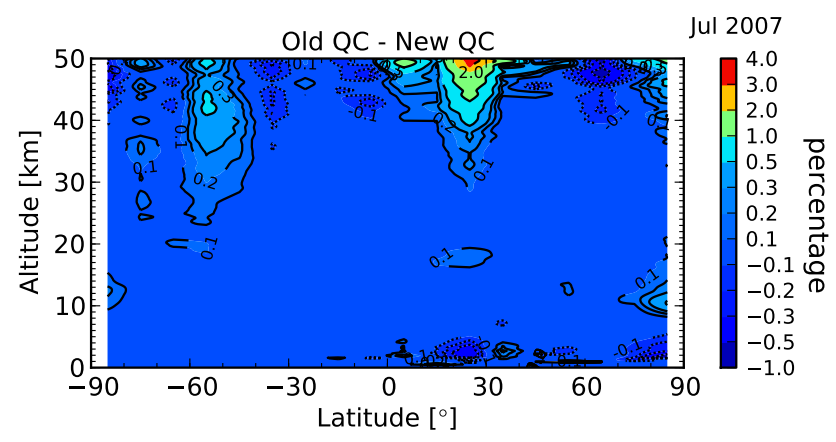

Figure 3. Relative difference between monthly mean refractivities obtained by average-profile inversion with standard quality control (old QC) and additional quality control (new QC) for CHAMP data from July 2007.

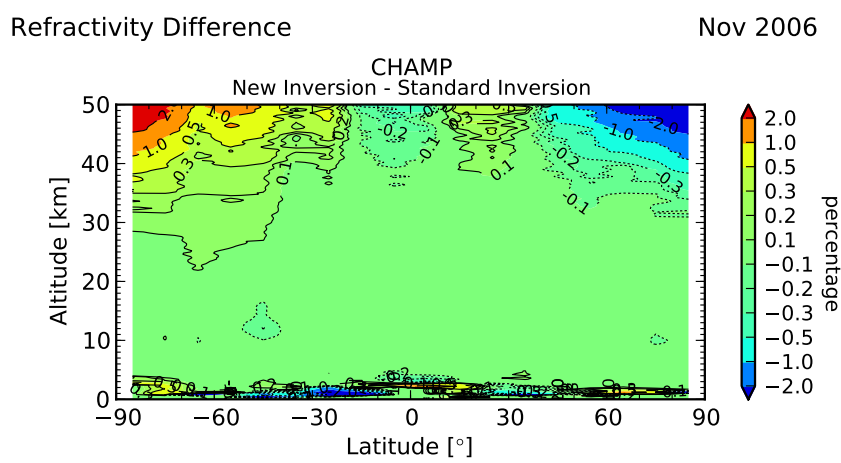

Figure 4. Relative difference between monthly mean refractivity from CHAMP data, using average-profile inversion and standard inversion, for November 2006

\subsection{Initial analysis}

In a first analysis the effect of applying an additional bending-angle QC on monthly mean refractivity profiles was studied. To answer this question, monthly mean refractivity profiles have been calculated with and without additional QC, using the API technique. Figure 3 shows the relative difference between monthly mean refractivities for CHAMP data from July 2007, calculated with "old QC" and with "new QC". "Old QC" refers to the standard QC at the DMI, applied to single profiles (Gorbunov et al., 2006); "new QC" refers to an additional outlier rejection at bending-angle level. Differences between the two cases below an altitude of $30 \mathrm{~km}$ are less than $|0.2| \%$; above $30 \mathrm{~km}$, areas of increased differences start to appear, showing, for the case of July 2007 , a value of up to $4 \%$ at an altitude of $50 \mathrm{~km}$. Investigating other months (not shown), the main impact of the additional QC is on the mid- to high latitudes. To further extend the quality control investigation, API-processed profiles with and without additional QC have been studied relative to colocated ECMWF profiles. In general profiles with additional QC showed smaller differences relative to colocated 

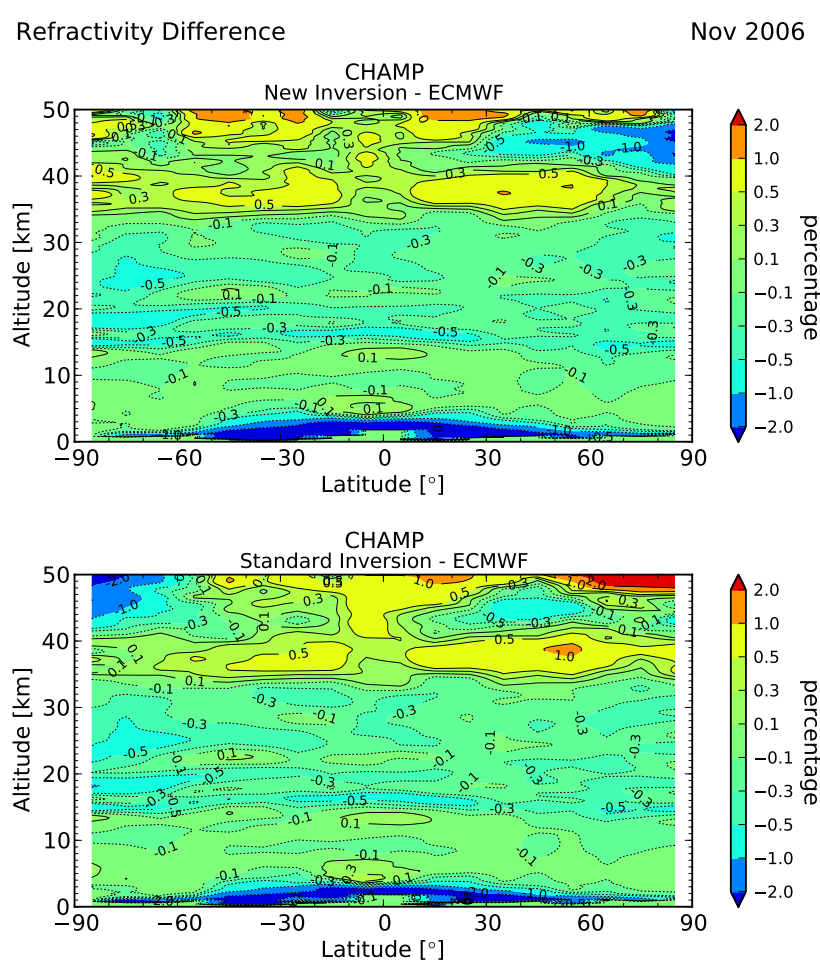

Figure 5. Relative difference between monthly mean refractivity from CHAMP data and from collocated ECMWF data, using average-profile inversion (top plot) and standard inversion (bottom plot), for November 2007.

ECMWF profiles than when using only the standard quality control. This analysis together with the results of the bending-angle study (see, e.g., Fig. 1) led to the decision to always apply an additional outlier rejection on bending-angle profiles in the case of CHAMP data.

Next, zonal monthly refractivities were compared, obtained from inverting averaged bending angles as well as statistically optimized single profiles. Figure 4 shows the result of the difference between the two methods for the month November 2006, based on CHAMP data. The relative differences are smaller than $|0.1| \%$ below altitudes of $30 \mathrm{~km}$, increase to about $|1| \%$ at $40 \mathrm{~km}$, and reach more than $\pm 2 \%$ at $50 \mathrm{~km}$ altitude and high latitudes. As a next step, in Fig. 5, the relative difference between the inversion method and the colocated ECMWF profile was analyzed. The top plot shows the results for the new inversion technique, while the bottom plot shows the results for the standard inversion. Below $35 \mathrm{~km}$, differences with respect to ECMWF are almost identical for the two inversion methods. This shows that, below $35 \mathrm{~km}$, statistical optimization did not cause any significant effects, due to the a priori information. Regarding the region between 35 to $50 \mathrm{~km}$, differences start to increase relative to ECMWF, with values larger than $\pm 2 \%$ at high latitudes for the standard inversion. For the new inversion, relative differences are smaller at high latitudes, compared to the standard retrieval. Figures 4 and 5 already hint at a known limitation of the current retrievals based on statistical optimization, showing larger biases during winter conditions at high latitudes and altitudes. The results suggest that, through the introduction of the rather complex statistical optimization, no obvious benefits are introduced in the generation of refractivity climatologies. Furthermore, performing a systematic analysis similar to Fig. 5 suggests that the API approach is feasible for both CHAMP and COSMIC data.

\subsection{Detailed comparison of new inversion to standard inversion}

One of the motivations for the introduction of the new retrieval scheme was to circumvent the rather complex SO and hence to remove a source of structural uncertainty from the processing. In Ao et al. (2012) and Gleisner and Healy (2013), first positive results of the API technique, which is designed for the study of climatologies, were shown for COSMIC satellite data. However, it has been questioned whether the API technique is also applicable to CHAMP data. The last section showed that average profile processing works for CHAMP data as well as long-term trend studies are possible. However, it needs to be evaluated whether the new retrieval scheme is equally good, or maybe even better, than the standard retrieval scheme. Hence, both processing methods need to be compared and studied on a longer timescale.

Due to the known limitations of the current retrievals at high latitudes, the main differences between the two processing methods are expected in that geographical region. Hence, Fig. 6 studies the relative differences between CHAMP and colocated ECMWF data for northern high latitudes, comparing the standard and API retrieval for the time period from September 2001 until September 2008. The results are very illuminating, since, for the standard retrieval, the plots clearly show maximal deviations from ECMWF for northern winter conditions. The standard retrieval shows a very distinct signal, occurring at polar-winter conditions and increasing with altitude and latitude. Also, the new retrieval exhibits a maximum increase in the relative differences in the winter months, but it is slightly reduced compared to the standard retrieval and less distinct. Nevertheless, a slight increase in the bias for the remaining non-winter months can be observed for the average bending-angle approach.

Finally, in Fig. 7 differences between RO satellite data and ECMWF analysis data for a midlatitude band and a lowlatitude band are studied, comparing the two processing methods. For the latitude band of 60 to $70^{\circ} \mathrm{N}$, the standard retrieval still shows a slightly more enhanced difference in the winter months. With decreasing latitudes, the relative differences become practically the same for the two processing methods.

Furthermore, we find a change of the seasonal pattern in 2006. The differences to the ECMWF decrease, which could be due to the change of the resolution of the ECMWF anal- 

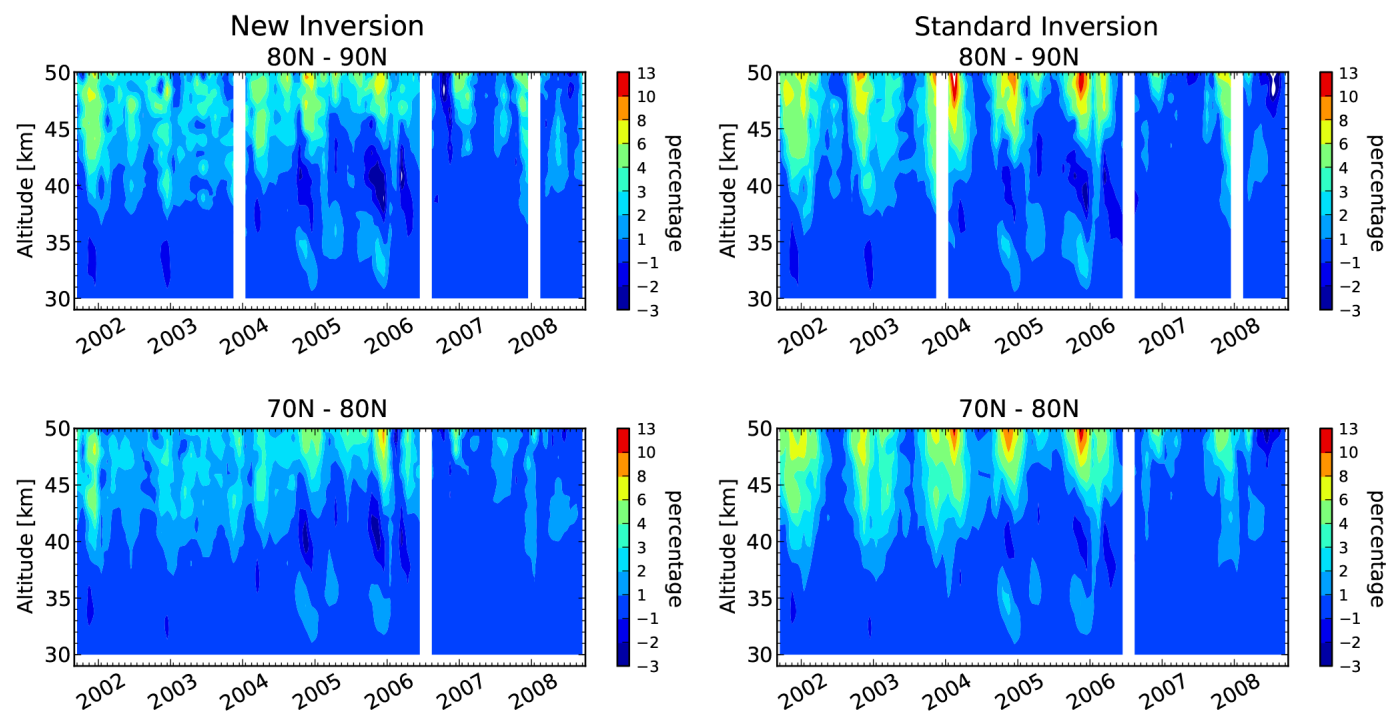

Figure 6. Relative differences between monthly mean refractivities from CHAMP data and from collocated ECMWF data, comparing new inversion (left) and standard inversion (right), from September 2001 until September 2008 for the zonal bins 80 to 90 and 70 to $80^{\circ} \mathrm{N}$.

ysis fields in February 2006 from 60 vertical levels up to 91 levels and the start of assimilating GPS RO data (December 2006) into the fields. This change in the generation of ECMWF data also affects the time series, leading to smaller differences predominately in the years 2007 and 2008.

A similar pattern of relative differences could be observed for the Southern Hemisphere, which is why it is not shown here. Figures 6 and 7 illustrated that main differences between the two processing methods arise exclusively at higher latitudes and altitudes. The seasonally varying differences relative to ECMWF for the standard retrieval are probably due to problems with the SO. The new approach shows smaller differences relative to ECMWF in high-latitude winter months but, however, also slightly larger differences in non-winter months. Further investigations will be necessary.

\section{Discussion and conclusions}

In this study the idea of forwarding bending-angle climatologies, instead of SO single bending-angle profiles through an Abel transformation was tested on CHAMP data. It has been shown that $\mathrm{SO}$ is a source of structural uncertainty between different processing centers (Steiner et al., 2013), which may be circumvented by averaging primarily in bending-angle space. An initial analysis of the average bending-angle approach has been performed on COSMIC satellite data (Ao et al., 2012; Gleisner and Healy, 2013). However, in order to obtain long-term climate data products, the use of CHAMP data is also required. Since the CHAMP satellite mission has a reduced number of occultations and an increased noise level compared to the COSMIC mission, it was questionable whether the approach would also be successful on CHAMP data.

The analysis was started by generating $10^{\circ}$ monthly zonal bending-angle profiles, studying bending-angle means and medians. Due to large-scale wiggles in the mean value, an additional bending-angle outlier rejection was introduced for values larger or smaller than $\pm 30 \mu$ rad between an altitude of 50 and $80 \mathrm{~km}$.

After performing the Abel transformation on the average bending-angle profiles, monthly zonal refractivities were studied, leading to the firm conclusion that the average bending-angle approach is also applicable to CHAMP data. Below an altitude of $35 \mathrm{~km}$, the standard single-profile processing and the average profile processing are almost identical, for CHAMP as well as for COSMIC satellite data. Above an altitude of $35 \mathrm{~km}$, differences between the two processing methods start to increase, showing largest differences at high altitudes and high latitudes.

In order to understand discrepancies better, and since it is known that retrievals based on SO may exhibit structural errors at high altitudes and in high-latitude winter conditions, a detailed analysis of this region was performed. Differences in monthly mean refractivities relative to colocated ECMWF data were studied on a longer timescale, detecting a clear signal of an increased bias relative to ECMWF at high latitudes in the wintertime for the standard retrieval. For the new retrieval, the bias was reduced, with, however, slightly larger deviations for non-winter months compared to the standard retrieval. Studying mid- to low latitudes, the two retrievals showed practically identical results relative to ECMWF, indicating a robustness in the choice of the approach in that geographical region. 

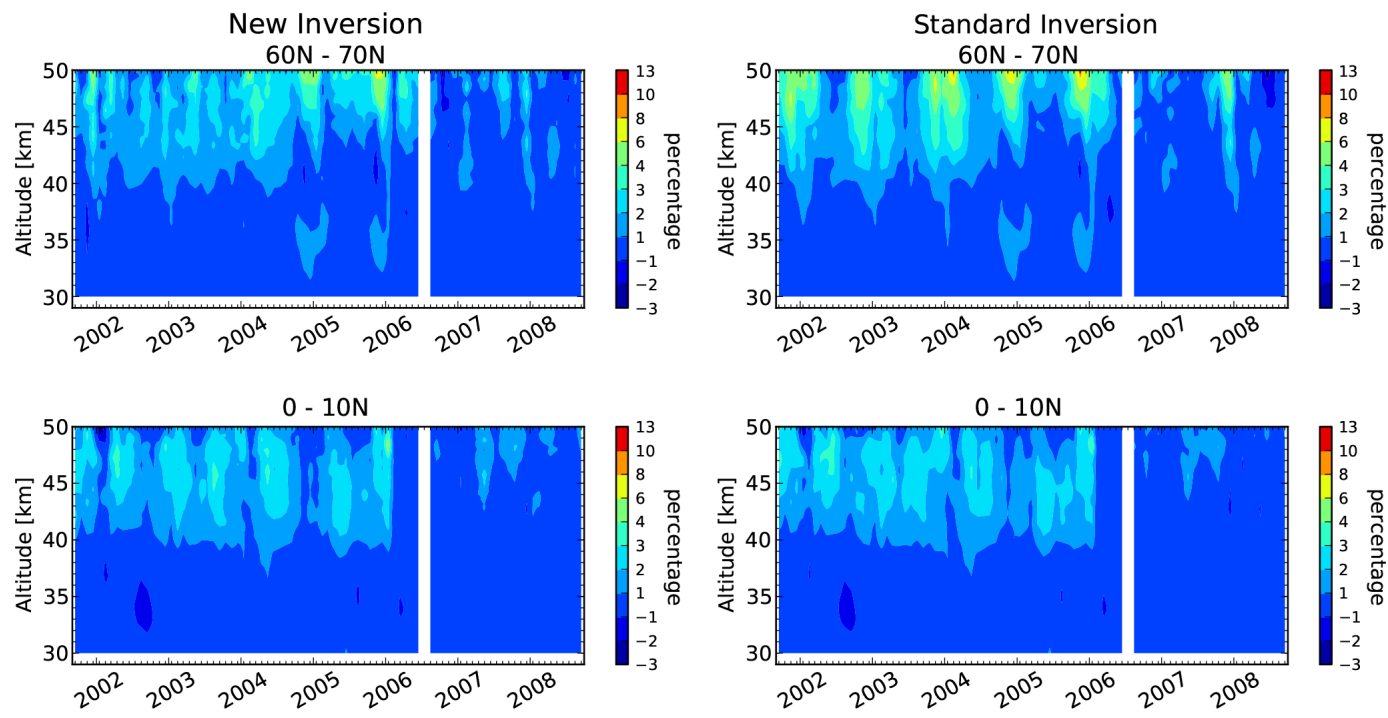

Figure 7. Relative differences between monthly mean refractivities from CHAMP data and from collocated ECMWF data, comparing new inversion (left) and standard inversion (right), for September 2001 until September 2008 and $10^{\circ}$ zonal bins for northern mid- to low latitudes.

For a correct interpretation of the results of Figs. 6 and 7, further investigations of the new averaging approach will be necessary by using, e.g., other reference data sets. Also, the impact of residual ionospheric errors needs to be analyzed, since observational data are used at higher altitudes. Although there is a general interest in addressing this problem, in the case of the averaging approach, the residual influence of the ionosphere needs to be understood. On the other hand, statistical optimization aims to reduce random errors; by using a priori information, it may partly mitigate that problem.

Nevertheless, for the application of climatologies, the new approach showed some very promising results. Since it is free of a priori information up to $80 \mathrm{~km}$ altitude, the API technique makes the error characterization of the resulting climate data products much easier. For the standard retrievals, used at different processing centers, it may be difficult to distinguish at what altitude the influence of the model data starts and when purely observational data is used. This depends on the implementation of the SO of the processing center. Hence, as a next step it would be interesting to study the API technique with the retrieval of different processing centers and compare the resulting climate data products. If the differences are very small, it means that the new approach shows the encouraging result of a robustness among processing centers.

Finally, we also suggest studying spatial and temporal limits of the approach. So far, $5^{\circ}$ zonal bins in COSMIC data and $10^{\circ}$ zonal bins in CHAMP and COSMIC data sets have been tested on a monthly basis. It would be interesting to test a further longitudinal binning, e.g., $60^{\circ} \times 10^{\circ}$ longitudinal $\times$ latitudinal bins, or to test a 2 -week timescale on zonal bins. If the averaging approach starts to fail for other binning choices, it means one is restricted to zonal climatologies with this special approach.

We conclude that it is possible to retrieve monthly refractivity profiles directly from averaged bending angles, using RO data from the CHAMP satellite mission. In that regard, the investigations showed very positive results, which opened the door to a valid way of circumventing the SO step in climatological studies. Further necessary investigations have been pointed out and discussed, which should clarify the limits and possibilities of the new approach.

Acknowledgements. We thank UCAR/CDACC for providing CHAMP and COSMIC excess phase data and the ECMWF for providing analysis data. Furthermore, we are grateful to ROM SAF, which made the visiting scientist activity possible during which the work was conducted. Finally, we thank Kent B. Lauritsen at the DMI for discussions and his support and Marc Schwärz at the WEGC for his technical support.

Edited by: A. von Engeln

\section{References}

Ao, C. O., Mannucci, A. J., and Kursinski, E. R.: Improving GPS radio occultation stratospheric refractivity retrievals for climate benchmarking, Geophys. Res. Lett., 39, L12701, doi:10.1029/2012GL051720, 2012.

Foelsche, U. and Scherllin-Pirscher, B.: Development of bending angle climatology from RO data, CDOP Visiting Scientist Report 14, DMI, Copenhagen, Denmark, 51 pp., available at: http: //www.romsaf.org, 2012.

Foelsche, U., Kirchengast, G., Steiner, A. K., Kornblueh, L., Manzini, E., and Bengtsson, L.: An observing system simulation 
experiment for climate monitoring with GNSS radio occultation data: setup and test bed study, J. Geophys. Res., 113, D11108, doi:10.1029/2007JD009231, 2008.

Gleisner, H. and Healy, S. B.: A simplified approach for generating GNSS radio occultation refractivity climatologies, Atmos. Meas. Tech., 6, 121-129, doi:10.5194/amt-6-121-2013, 2013.

Gobiet, A. and Kirchengast, G.: Advancements of Global Navigation Satellite System radio occultation retrieval in the upper stratosphere for optimal climate monitoring utility, J. Geophys. Res., 109, D24110, doi:10.1029/2004JD005117, 2004.

Gorbunov, M.: Canonical transform method for processing radio occultation data in the lower troposphere, Radio Sci., 37, 1076, doi:10.1029/2000RS002592, 2002.

Gorbunov, M., Lauritsen, K., Rhodin, A., Tomassini, M., and Kornblueh, L.: Radio holographic filtering, error estimation, and quality control of radio occultation data, J. Geophys. Res., 111, D10105, doi:10.1029/2005JD006427, 2006.

Ho, S.-P., Kirchengast, G., Leroy, S., Wickert, J., Mannucci, A. J., Steiner, A. K., Hunt, D., Schreiner, W., Sokolovskiy, S., Ao, C., Borsche, M., von Engeln, A., Foelsche, U., Heise, S., Iijima, B., Kuo, Y.-H., Kursinski, E. R., Pirscher, B., Ringer, M., Rocken, C., and Schmidt, T.: Estimating the uncertainty of using GPS radio occultation data for climate monitoring: intercomparison of CHAMP refractivity climate records from 2002 to 2006 from different data centers, J. Geophys. Res., 114, D23107, doi:10.1029/2009JD011969, 2009.

Ho, S.-P., Hunt, D., Steiner, A. K., Mannucci, A. J., Kirchengast, G., Gleisner, H., Heise, S., von Engeln, A., Marquardt, C., Sokolovskiy, S., Schreiner, W., Scherllin-Pirscher, B., Ao, C., Wickert, J., Syndergaard, S., Lauritsen, K. B., Leroy, S., Kursinski, E. R., Kuo, Y.-H., Foelsche, U., Schmidt, T., and Gorbunov, M.: Reproducibility of GPS radio occultation data for climate monitoring: profile-to-profile inter-comparison of CHAMP climate records 2002 to 2008 from six data centers, J. Geophys. Res., 117, D18111, doi:10.1029/2012JD017665, 2012.
Kursinski, E. R., Hajj, G. A., Schofield, J. T., Linfield, R. P., and Hardy, K. R.: Observing Earth's atmosphere with radio occultation measurements using the Global Positioning System, J. Geophys. Res., 102, 23429-23465, doi:10.1029/97JD01569, 1997.

Lauritsen, K. B., Syndergaard, S., Gleisner, H., Gorbunov, M. E., Rubek, F., Sørensen, M. B., and Wilhelmsen, H.: Processing and validation of refractivity from GRAS radio occultation data, Atmos. Meas. Tech., 4, 2065-2071, doi:10.5194/amt-4-2065-2011, 2011.

Lohmann, M. S.: Application of dynamical error estimation for statistical optimization of radio occultation bending angles, Radio Sci., 40, RS3011, doi:10.1029/2004RS003117, 2005.

Scherllin-Pirscher, B.: Further development of BAROCLIM and implementation in ROPP, ROM SAF CDOP-2 Visiting Scientist Report 19, Ref: SAF/ROM/DMI/REP/VS19/001, 56 pp., available at: http://www.romsaf.org, 2013.

Scherllin-Pirscher, B., Syndergaard, S., Foelsche, U., and Lauritsen, K. B.: Generation of a Bending Angle Radio Occultation Climatology (BAROCLIM) and its use in radio occultation retrievals, Atmos. Meas. Tech. Discuss., 7, 8193-8231, doi:10.5194/amtd7-8193-2014, 2014.

Steiner, A. K., Hunt, D., Ho, S.-P., Kirchengast, G., Mannucci, A. J., Scherllin-Pirscher, B., Gleisner, H., von Engeln, A., Schmidt, T., Ao, C., Leroy, S. S., Kursinski, E. R., Foelsche, U., Gorbunov, M., Heise, S., Kuo, Y.-H., Lauritsen, K. B., Marquardt, C., Rocken, C., Schreiner, W., Sokolovskiy, S., Syndergaard, S., and Wickert, J.: Quantification of structural uncertainty in climate data records from GPS radio occultation, Atmos. Chem. Phys., 13, 1469-1484, doi:10.5194/acp-13-1469-2013, 2013. 